\title{
DEADLOCK ANALYSIS IN FMS IN THE PRESENCE OF FLEXIBLE PROCESS PLANS - A SIMULATION STUDY
}

\author{
Jain, A. ; Jain, P. K.* \& Singh, I. P.* \\ *Mechanical Engineering Department, National Institute of Technology, Kurukshetra, 136119, India \\ E-mail: ajaijain12@indiatimes.com \\ ** Mechanical \& Industrial Engineering Department, Indian Institute of Technology, \\ Roorkee, 247667, India \\ E-mail: pjainfme@iitr.ernet.in
}

\begin{abstract}
The increased use of FMS to provide customers with diversified products efficiently has created a significant set of operational challenges for managers. This technology presents a number of decision problems to be solved by researchers and practitioners. There have been a number of attempts to solve design and operational problems in FMS. A special attention has been given to batch scheduling when flexible process plans (FPPs) remain available on the shop floor. An extensive simulation study under two manufacturing environments is performed to study the system performance in the presence of FPPs. Several interesting conclusions have been drawn from deadlock, makespan and mean flow time criteria.
\end{abstract}

Key Words: FMS Scheduling, Flexible Process Plans, Deadlock Avoidance

\section{INTRODUCTION}

In recent years there has been considerable interest in the developments of control theory and methods for design, scheduling operations and performance evaluation of flexible manufacturing systems (FMSs). FMSs combine the efficiency of mass production and flexibility of a job shop to best suit the batch production of mid-volume and mid-variety of products. The flexibility of an FMS is mainly due to the capability of performing different operations within the same station and material handling system, which provides fast and flexible transfer of parts with in the system. Since FMSs are capital intensive, an effective management and control system is needed for their successful implementation.

For smooth operation of an FMS, there are several problems such as aggregate planning, resource grouping, disaggregate planning and scheduling that are to be solved in a hierarchical manner. Among them, scheduling has been researched over past several years and it is concerned with decisions that have to be taken when the system is in operation. FMS scheduling is quite different from that in conventional job shop because of its integrated hardware and its operating conditions such as (1) presence of flexible (multiple) process plans (FPPs), (2) limited buffer space availability, (3) considerations of transportation time and transportation capacity, (4) deterministic processing times, (5) reduced set-up time between consecutive operations (6) limited pallet and fixtures availability and (7) considerations of constraints on tool changing facilities [1]. These factors make most FMS scheduling problems NP-hard as they become intractable and complex [2]. Thus real time scheduling approach is advocated as appropriate approach for FMS scheduling problems by many researchers [3, 4].

In FMS, flexible process plans (FPPs) are common due to the presence of versatile machines that provide alternatives for adapting to dynamic environment and improve system performance [5]. FPPs can be generated with the consideration of operation flexibility (possibility of performing an operation on more than a machine), sequencing flexibility (possibility of interchanging the sequence in which required manufacturing operations are 
performed) and processing flexibility (possibility of producing the same manufacturing feature with alternative operations, or sequence of operations) [6]. It is important to mention that a computer controlled flexible automation system is essential to implement FPPs in practice [7].

In FMSs/automated manufacturing systems (AMSs), deadlock free operation is an essential control requirement. A deadlock is a situation where each of a set of two or more parts keeps waiting indefinitely for the other parts in the set to release resources. Deadlock occurs when various parts with different routings compete for finite number of resources. When a deadlock occurs in a manufacturing system, part flow is inhibited, no activity/event occurs in the system and system comes to a stand still. [8] stated that a deadlock occurs if and only if the following four conditions exist in the system simultaneously: mutual exclusion, hold and wait, no pre-emption and circular wait. Resolving a deadlock thus amounts to relaxing at least one of these conditions. In manufacturing systems, the first three conditions are generally satisfied. Thus only the fourth condition determines whether a deadlock occurs [8]. Therefore, if system controller can predict the occurrence of circular wait, deadlock can be avoided.

This paper, initially, provides a literature review of manufacturing research on deadlock and FPPs. Subsequently, an extensive simulation study is carried out using Petri nets (PN) model of an example FMS, to study the system performance in two manufacturing environments viz., VBM (virtual batch manufacturing) and VLM (virtual line manufacturing) from deadlock, makespan and mean flow time viewpoints. VBM represents the manufacturing scenario in which operation times are higher and not comparable with transportation times while VLM represents the manufacturing scenario in which operation times are comparable with transportation times.

\section{LITERATURE REVIEW}

This section provides a review of relevant literature in the areas of deadlock and FPPs. Review of literature indicates that two approaches are advocated for eliminating deadlocks in FMS/AMS. One emphasizes on designing an inherently deadlock free manufacturing system $[9,10,11]$ while other approach eliminates possible deadlocks through proper operational control [12, 13, 14, 15]. Researcher community advocated following three strategies for eliminating the deadlock occurrence through operational control approach: deadlock prevention, deadlock detection/recovery and deadlock avoidance. Deadlock prevention is a simple approach in which predetermined rules are followed that ensures that at least one of the four necessary conditions does not hold. In case of a manufacturing system, system controller must determine in advance all possible routes of the parts in the manufacturing system to prevent occurrence of deadlock. Thus this approach is based on static resource allocation policy and easy to implement but results in poor resource utilization as well as system throughput. Deadlock detection/recovery strategy allows the system to enter a deadlock state and then initiate system recovery. It can be an expensive strategy since it might involve removal of some semi-finished parts from system or resource preemption [16]. Deadlock avoidance is a more practical approach for real-world manufacturing system [17]. It refers to dynamic resource allocation policies and examines the system's state dynamically and by passes deadlock state carefully, in real time, resulting in better resource utilization and system throughput. Deadlock prediction is an essential part of the deadlock avoidance procedure to ascertain the locations of deadlocks in advance. In general, a system with dynamic routings require an algorithm to predict and avoid deadlocks in real-time. It is important to mention that deadlock resolution algorithms are dependent on system configuration and the way the system operates [15]. Several deadlock avoidance policies 
(DAP) such as Banker's algorithm [18, 19], resource upstream neighborhood (RUN) [20, 21], resource order (RO) [22] have been developed by various researchers. However, authors found only a single paper that addresses the issue of DAP in the presence of flexible routing (operations flexibility) [23].

For FPPs and FMS scheduling, [24, 25] cover the published work in this area and this review includes only important published papers. [26] studied the impact of varying level of routing flexibilities on the makespan of a FMS and concluded that increase in routing flexibility, when made at the cost of an associated penalty on operation processing time is not always beneficial. There is an optimal flexibility level beyond which system performance deteriorates. [27] developed and evaluated computationally efficient procedures for scheduling jobs in a large-scale manufacturing system in the presence of FPPs for maximum lateness performance measure and concluded that (i) there are substantial differences in schedule performance between scheduling with alternative and scheduling without alternatives. Scheduling with alternatives can greatly improve the ability to satisfy due-date under various shop floor conditions and (ii) there are substantial differences in schedule performance between scheduling with alternative routing, operation and sequencing. Scheduling with alternative operations had the largest schedule improvement and scheduling with alternative sequences had the smallest schedule improvement. [28] developed a simulation software (OOSimFlex) that is capable of modeling different alternative systems design and analyzing a manufacturing system using multiple performance measures under different manufacturing flexibility levels. [29] carried out scheduling of FPPs in a mould manufacturing shop instead of FMS using branch and bound procedure to optimize priority weighted earliness of jobs. [30] used genetic algorithm for FMS scheduling/rescheduling and observed that routing flexibility has a significant impact in case of disruptions (machine breakdown, rush order arrival, order cancellation and increased order priorities). Literature review on dispatching rules is beyond the scope of this paper. [31] reported that in FMS, PT2 (smallest ratio of processing time to number of remaining operations), MOPNR (most operations remaining), MWR (most work remaining) and PWR (smallest ratio of processing time to remaining work) rules perform better than other dispatching rules for makespan. [25] proposed four dispatching rules (\%CW, CW1, CW2, CW5) and presented several interesting conclusions on performance of dispatching rules.

Literature review reveals there are several studies to assess the impact of flexibility on system performance but few studies have been conducted by researcher community to study the system performance with FPPs for identifying various deadlock avoidance policies. The objective of the present paper is to study the system performance from deadlock viewpoint in the presence of FPPs that will assist operations managers to device deadlock avoidance algorithms. This paper also assesses the system performance from makespan and mean flow time performance measures in order to identify variables affecting them.

\section{SYSTEM DESCRIPTION AND MODEL CHARACTERISTICS}

The FMS considered in the present study is shown in Fig. 1 [24]. This system consists of four machines (M1, M2, M3, and M4) with work-in-process buffers, two robots, system input (BIN) and output (B-OUT) buffers and a load/unload (L/UL) station. Each machine has individual input and output buffer of capacity three and two respectively. A giant robot R-2 serves all the four machines. It is assumed that L/UL station is of infinite capacity and BIN/B-OUT have a capacity of ten and eight respectively. Another robot R-1 operates between L/UL station and B-IN/B-OUT for part transfer. Travel times of R-1 and R-2 while serving other system resources are given in Table I [24]. 


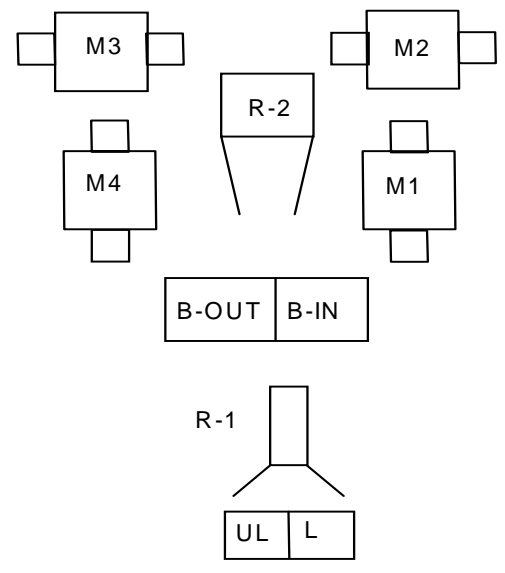

Figure 1: Taken FMS configuration [24].
Table I: R-1 and R-2 unit travel times [24].

\begin{tabular}{|l|l|l|l|l|l|l|l|}
\hline & & $\begin{array}{c}\text { L/ } \\
\text { UL }\end{array}$ & $\begin{array}{c}\text { B-IN/ } \\
\text { B- } \\
\text { OUT }\end{array}$ & M1 & M2 & M3 & M4 \\
\hline \multirow{3}{*}{$\begin{array}{c}\text { Robot } \\
\text { R-1 }\end{array}$} & L/UL & 0 & 2 & - & - & - & - \\
\cline { 2 - 9 } & $\begin{array}{l}\text { B-IN/ } \\
\text { B-OUT }\end{array}$ & 2 & 0 & - & - & - & - \\
\hline \multirow{4}{*}{$\begin{array}{c}\text { Robot } \\
\text { R-2 }\end{array}$} & $\begin{array}{l}\text { B-IN/ } \\
\text { B-OUT }\end{array}$ & - & 0 & 3 & 5 & 5 & 3 \\
\cline { 2 - 9 } & M1 & - & 3 & 0 & 3 & 5 & 7 \\
\cline { 2 - 9 } & M2 & - & 5 & 3 & 0 & 3 & 5 \\
\cline { 2 - 9 } & M3 & - & 5 & 5 & 3 & 0 & 3 \\
\cline { 2 - 9 } & M4 & - & 3 & 7 & 5 & 3 & 0 \\
\hline
\end{tabular}

Legend: - Not Applicable.

The occurrence of manufacturing activity in the FMS is as follows. Initially, a raw part is loaded onto pallet at load station by manual operation. The palletized part is transported by R1 to B-IN. R-2 then moves parts from B-IN to various machines according to their process plans. When FPPs are available, a part can switch over to alternate process plan to overcome the non-availability of machine due to limited buffer space. The sequence of part flow at a machine is: machine input buffer $\rightarrow$ machine table $\rightarrow$ machine output buffer. When a part is completed, R-2 transports it back to B-OUT. R-1 again transports finished parts from B-OUT to unload station, where part is unloaded from the pallet manually. This empty pallet is again loaded with raw part of same part type, if available, and sent into the system. At any time, three-job types viz., raw jobs, semi-finished jobs and finished jobs are circulating and compete for the same resources (machines/robots). Thus, several operational decisions such as part selection decision (at B-IN, B-OUT, input and output buffer of each machine) and machine selection decision (at B-IN and output buffer of each machine in case of FPPs) are required for the smooth functioning of the FMS. These decisions are not described here for want of space and discussed in [24, 25]. Assumptions such as (i) operations and transportation times are deterministic and known in advance, (ii) each machine is continuously available for processing jobs, (iii) part preemption is not allowed, (iv) pallet and fixture availability is limited and it can load one part at the most, (v) All parts are available for processing at the start, although part entry into the system is dependent on the pallet availability and (vi) number of pallets available for each part type is in same proportion of the total pallets available as the proportion of their required production quantity with respect to the total production requirement that are in line with previous studies have been made in this work.

The FMS is modeled using Petri nets (PN) modeling approach and execution code is written in $\mathrm{C}$ programming language under Windows environment. The PN model of the example FMS is large and not included for want of space. However, a simplified version of PN model in the form of flow process diagram is shown in Fig. 2. This diagram depicts the information and material flow in the system. Part loading/unloading, part transfer and operations are pictured as circles. Waiting time and delays are same and pictured as letter D. Delays occur due to non-availability of machines and/or material handling equipment. When machines and/or material handling equipment are available, waiting time/delay is zero. All decision blocks are represented by diamonds and play a crucial role in regulating the flow of material and information in the manufacturing system. Here, D1 and D3 represent part selection at load station and B-IN respectively. Similarly, D5, D8, and D11 represent part election at M1, M2, and M3 respectively. A brief discussion of nodes is given in Table II. 


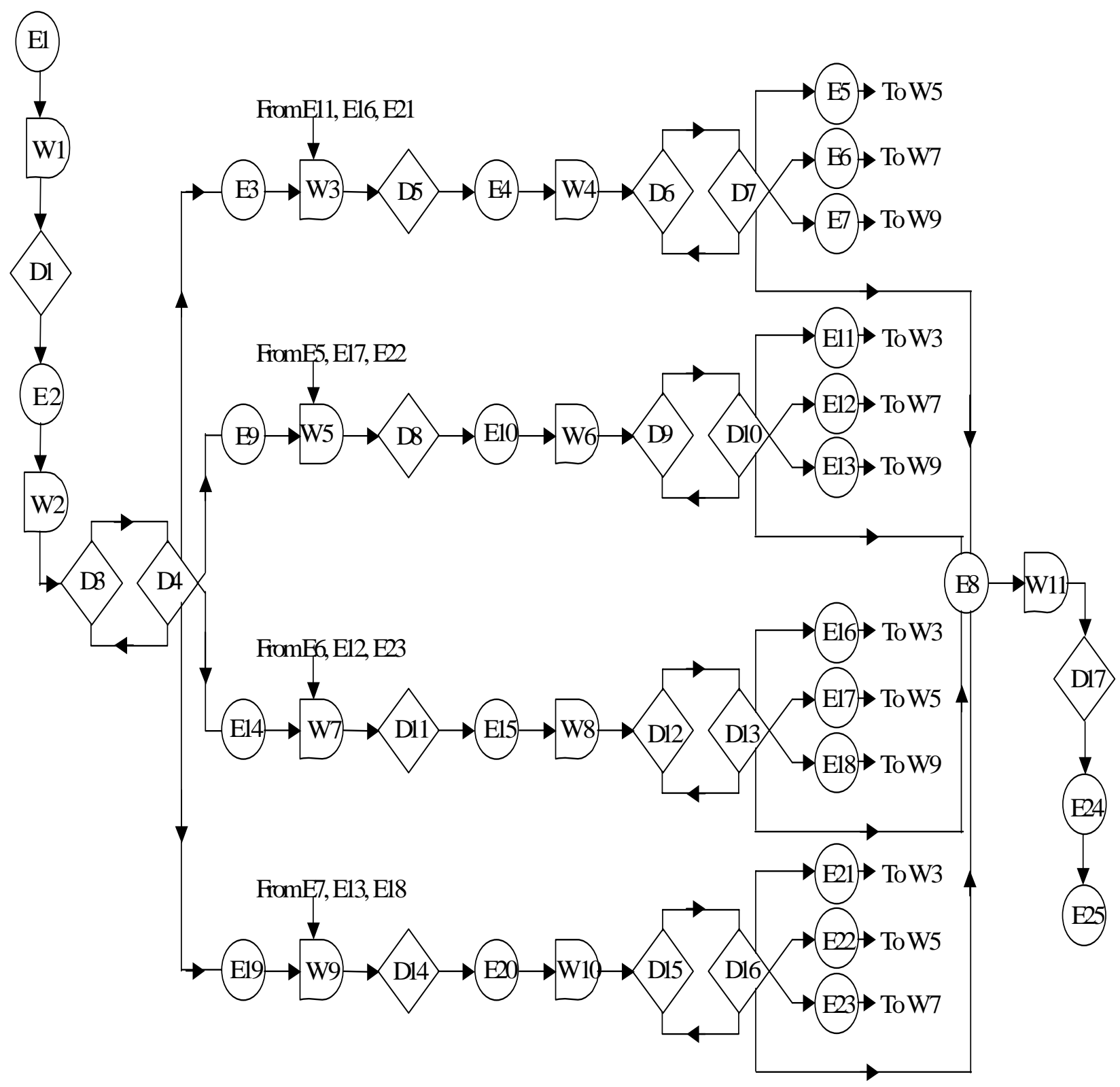

Figure 2: Process diagram of system controller.

\section{EXPERIMENTAL INVESTIGATIONS}

Experiments are designed and conducted by simulating the PN model of FMS in two manufacturing environments viz., VBM and VLM. Table III shows the flexible process plans of various part types that are considered in the present study. A production order belongs to either of manufacturing environment i.e. VBM or VLM. The number of pallets released to the system is taken as variable and it varies from six (30\% of work-in-process) to twenty (100\% of work-in-process). The various variables with their range/values considered are summarized in Table IV. 
Table II: Description of nodes of system flow diagram.

\begin{tabular}{|c|c|}
\hline Node & Description \\
\hline D1 & Part selection at load station \\
\hline D3 & Part selection at B-IN \\
\hline D4 & Machine selection at B-IN \\
\hline D5 & Part selection at input buffer of machine 1 \\
\hline D6 & Part selection at output buffer of machine 1 \\
\hline D7 & Machine selection at output buffer of machine 1 \\
\hline D8 & Part selection at input buffer of machine 2 \\
\hline D9 & Part selection at output buffer of machine 2 \\
\hline D10 & Machine selection at output buffer of machine 2 \\
\hline D11 & Part selection at input buffer of machine 3 \\
\hline D12 & Part selection at output buffer of machine 3 \\
\hline D13 & Machine selection at output buffer of machine 3 \\
\hline D14 & Part selection at input buffer of machine 4 \\
\hline D15 & Part selection at output buffer of machine 4 \\
\hline D16 & Machine selection at output buffer of machine 4 \\
\hline D17 & Part selection at B-OUT \\
\hline E1 & Part loading on pallet \\
\hline E2 & Part transfer to B-IN (by R-1) \\
\hline E3 & Part loading in input buffer of machine 1 (by R-2) \\
\hline E4 & Part processing on machine 1 \\
\hline E5 & Part transfer to input buffer of machine 2 (by R-2) \\
\hline E6 & Part transfer to input buffer of machine 3(by R-2) \\
\hline E7 & Part transfer to input buffer of machine 4 (by R-2) \\
\hline E8 & Part transfer to B-OUT (by R-2) \\
\hline E9 & Part loading in input buffer of machine 2 (by R-2) \\
\hline E10 & Part processing on machine 2 \\
\hline E11 & Part transfer to input buffer of machine 1 (by R-2) \\
\hline E12 & Part transfer to input buffer of machine 3(by R-2) \\
\hline E13 & Part transfer to input buffer of machine 4(by R-2) \\
\hline E14 & Part loading in input buffer of machine 3 (by R-2) \\
\hline E15 & Part processing on machine 3 \\
\hline E16 & Part transfer to input buffer of machine 1 (R-2) \\
\hline E17 & Part transfer to input buffer of machine 2 (R-2) \\
\hline E18 & Part transfer to input buffer of machine 4 (R-2) \\
\hline E19 & Part loading in input buffer of machine 4 (by R-2) \\
\hline E20 & Part processing on machine 4 \\
\hline E21 & Part transfer to input buffer of machine 1 (by R-2) \\
\hline E22 & Part transfer to input buffer of machine 2 (by R-2) \\
\hline E23 & Part transfer to input buffer of machine 3 (by R-2) \\
\hline E24 & Part transfer to unload station (by R-1) \\
\hline E25 & Part unloading from pallet \\
\hline W1 & Part at load station \\
\hline W2 & Part at B-IN \\
\hline W3 & Part in input buffer of machine 1 \\
\hline W4 & Part in output buffer of machine 1 \\
\hline W5 & Part in input buffer of machine 2 \\
\hline W6 & Part in output buffer of machine 2 \\
\hline W7 & Part in input buffer of machine 3 \\
\hline W8 & Part in output buffer of machine 3 \\
\hline W9 & Part in input buffer of machine 4 \\
\hline W10 & Part in output buffer of machine 4 \\
\hline W11 & Part in B-OUT \\
\hline
\end{tabular}


Table III: Process plans of various part types.

Table IV: Parameters and their range considered in the present work.

\begin{tabular}{|c|c|c|}
\hline $\begin{array}{c}\text { Part } \\
\text { Type } \\
\text { Number }\end{array}$ & $\begin{array}{c}\text { VLM -FPP } \\
M_{i}\left(T_{I}\right)\end{array}$ & $\begin{array}{c}\text { VBM -FPP } \\
M_{i}\left(T_{I}\right)\end{array}$ \\
\hline 1. & $\begin{array}{l}1(4)-3(7)-1(3)-4(2) \\
1(4)-3(7)-2(5)-4(2) \\
2(6)-3(7)-1(3)-4(2) \\
2(6)-3(7)-2(5)-4(2)\end{array}$ & $\begin{array}{l}1(60)-3(75)-2(60)-4(30) \\
1(60)-3(75)-1(58)-4(30) \\
2(65)-3(75)-2(60)-4(30) \\
2(65)-3(75)-1(58)-4(30)\end{array}$ \\
\hline 2. & $\begin{array}{l}2(4)-1(3)-2(2)-4(10) \\
2(4)-1(3)-2(2)-1(12) \\
2(4)-1(3)-3(3)-4(10) \\
3(7)-1(3)-2(2)-4(10)\end{array}$ & $\begin{array}{l}2(60)-1(45)-2(41)-4(90) \\
2(60)-1(45)-3(45)-4(90) \\
3(65)-1(45)-2(41)-4(90) \\
3(65)-1(45)-3(45)-4(90)\end{array}$ \\
\hline 3. & $\begin{array}{l}1(5)-4(2)-3(2)-2(3) \\
1(5)-3(2)-4(3)-2(3) \\
1(5)-2(4)-3(2)-2(3) \\
1(5)-2(4)-4(3)-2(3)\end{array}$ & $\begin{array}{l}1(60)-4(45)-3(40)-2(30) \\
1(60)-3(41)-4(45)-2(30) \\
1(60)-3(41)-2(45)-4(33) \\
1(60)-4(45)-2(45)-1(32)\end{array}$ \\
\hline 4. & $\begin{array}{l}2(3)-1(6)-2(5)-1(3) \\
2(3)-1(6)-2(5)-3(4) \\
3(6)-4(5)-2(5)-1(3) \\
3(6)-1(6)-2(5)-1(3)\end{array}$ & $\begin{array}{l}1(60)-2(82)-3(100)-2(100) \\
1(60)-4(80)-3(100)-2(100) \\
3(65)-2(82)-1(98)-4(100) \\
3(65)-4(80)-1(98)-4(100)\end{array}$ \\
\hline 5. & $\begin{array}{l}4(4)-3(4)-2(4)-4(8) \\
2(5)-1(3)-2(4)-4(8) \\
2(5)-3(4)-2(4)-4(8) \\
2(5)-1(3)-3(8)-4(8)\end{array}$ & $\begin{array}{l}4(97)-3(54)-2(40)-1(99) \\
4(97)-1(50)-3(42)-4(100) \\
2(100)-3(54)-2(40)-1(99) \\
2(100)-1(50)-3(42)-4(100)\end{array}$ \\
\hline 6. & $\begin{array}{l}4(6)-2(3)-3(2)-1(3) \\
3(7)-2(3)-3(2)-1(3) \\
4(6)-2(3)-3(2)-2(4) \\
4(6)-2(3)-4(2)-1(3)\end{array}$ & $\begin{array}{l}2(60)-1(100)-2(100)-3(80) \\
2(60)-1(100)-4(97)-3(80) \\
3(63)-4(100)-2(100)-1(78) \\
3(63)-4(100)-2(100)-3(80)\end{array}$ \\
\hline 7. & $\begin{array}{l}3(4)-4(4)-3(5)-4(5) \\
1(3)-2(5)-3(5)-2(6) \\
1(3)-4(4)-3(5)-4(5) \\
3(4)-2(5)-3(5)-4(5)\end{array}$ & $\begin{array}{l}4(57)-1(95)-2(62)-4(40) \\
4(57)-1(95)-2(62)-1(42) \\
1(60)-2(100)-3(60)-4(40) \\
1(60)-2(100)-3(60)-1(42)\end{array}$ \\
\hline Legend: & $\mathrm{M}_{\mathrm{i}}$ - Machine Number & $\mathrm{T}_{\mathrm{i}}$ - Operation Time \\
\hline
\end{tabular}

\begin{tabular}{|c|c|c|}
\hline $\begin{array}{l}\text { S. } \\
\text { No. }\end{array}$ & Parameter & $\begin{array}{l}\text { Range/Value } \\
\text { Employed }\end{array}$ \\
\hline 1. & $\begin{array}{l}\text { Production quantity of each } \\
\text { part type in a production } \\
\text { order }\end{array}$ & $20-50$ \\
\hline 2. & $\begin{array}{l}\text { Number of part types in a } \\
\text { production order }\end{array}$ & $3-4$ \\
\hline 3. & Number of operations per job & 4 \\
\hline 4. & $\begin{array}{l}\text { Manufacturing } \quad \text { system } \\
\text { environment }\end{array}$ & VBM and VLM \\
\hline 5. & Transportation time & 2-7 units \\
\hline 6. & Operation time & $\begin{array}{l}\text { 2-15 units (for VLM } \\
\text { environment) and } \\
20-100 \text { units (for VBM } \\
\text { environment) }\end{array}$ \\
\hline 7. & $\begin{array}{l}\text { Number of pallets released to } \\
\text { the system }\end{array}$ & $\begin{array}{l}6-20 \text { in steps of } 2 \text { i.e. } 6 \\
8,10,12,14,16,18 \\
20\end{array}$ \\
\hline 8. & Dispatching rule & $\begin{array}{l}\text { SPT, PT2, \%CW, } \\
\text { CW1, CW2, CW5 }\end{array}$ \\
\hline 9. & Performance measure & $\begin{array}{l}\text { Makespan, mean flow } \\
\text { time }\end{array}$ \\
\hline
\end{tabular}

Six case studies in each manufacturing environment (total twelve case studies) are considered. Figs. 3 and 4 show variation of makespan and mean flow time with $n p$ for case study 1 (in VBM) and Figs. 5 and 6 show the above variations for case study 7 (in VLM). For other case studies, results are summarized in Tables V and VI for VBM and VLM respectively.

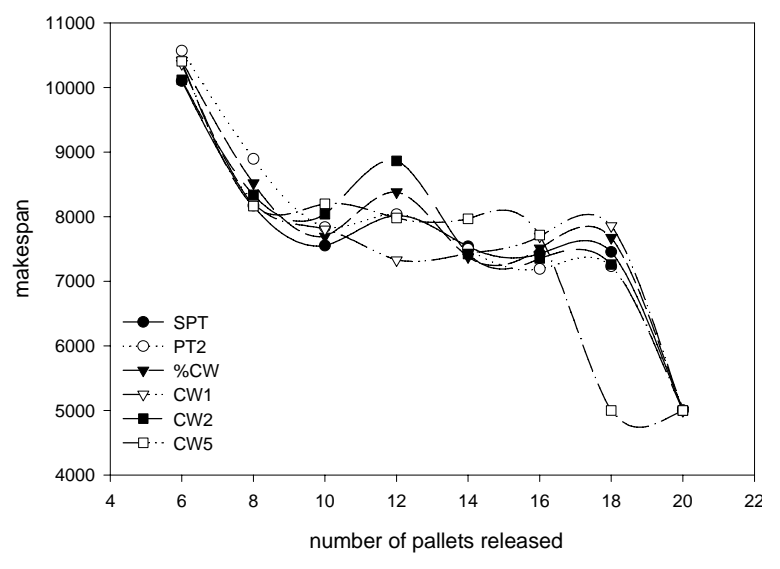

Fig. 3 Variation of Makespan with np (VBM) (5000 shows deadlock state)

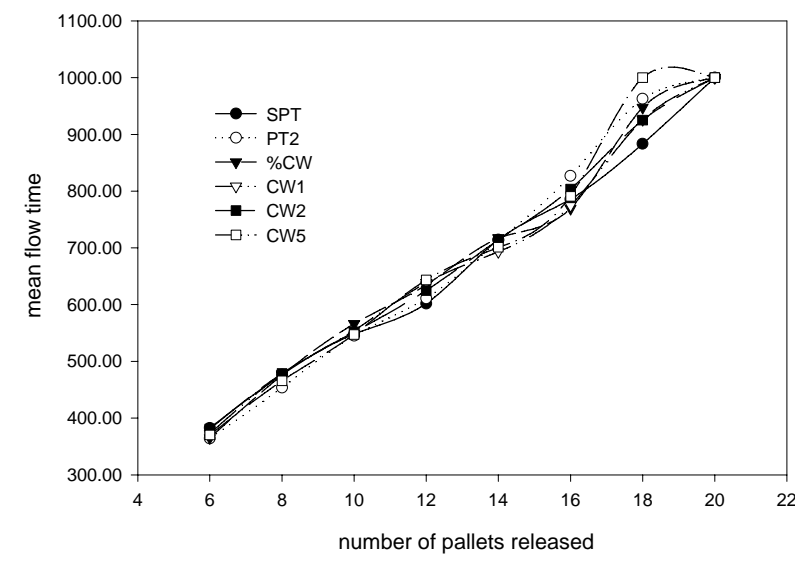

Fig 4 Variation of Mean Flow Time with np (VBM) (1000 shows deadlock state)

Table V shows that for case study 2 in VBM at $n p=16$, system reaches to deadlock state for PT2 and CW1 rules and other rules do not give rise to deadlock state at $n p=16$. Similarly, the Table V shows that for case study 2 in VBM at $n p=16$, system reaches to deadlock state 
for PT2 and CW1 rules and other rules do not give rise to deadlock state at $n p=16$. Similarly, at $n p=18$, deadlock state of system is reached for SPT, PT2, CW1, CW2 and CW5 rules and other rule i.e. \%CW does not give rise to deadlock state of system (at $n p=18$ ). For case study 4 at $n p=14$, CW2 give rise to deadlock state while other rules avoid it i.e. for a given production order and $n p$, deadlock state of system can be avoided by changing the dispatching rule. This observation is also true for other case studies in VBM (Table V and Fig. 3). This phenomenon may be attributed to the fact that during FMS scheduling, at B-IN and output buffer of each machine, part selection is performed using a dispatching rule. The selected part may be different due to different DRs at these stations and it may happen that the selected parts due to one DR can't be allocated to any machine according to their flexible process plans and system reaches to deadlock state. Thus, it can safely be concluded that for a given production order and $n p$, deadlock state of system can be avoided by changing the DR.

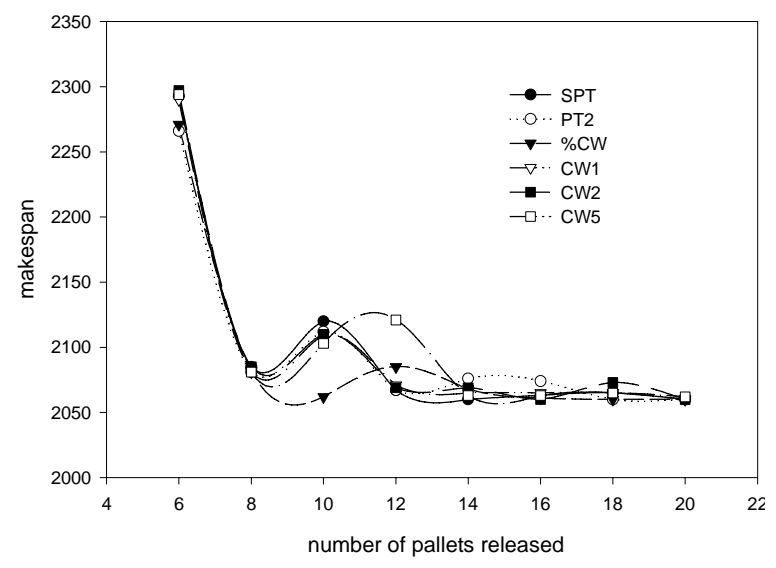

Fig. 5 Variation of Makespan with $n p(V L M)$

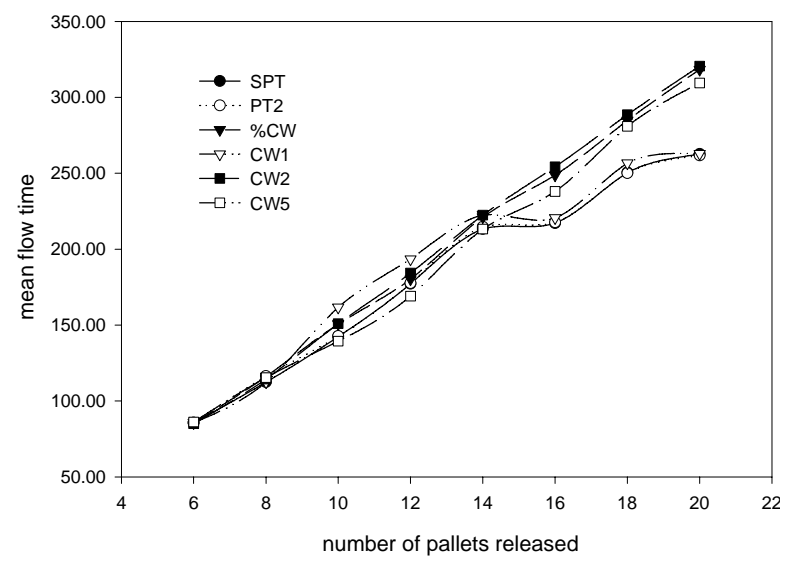

Fig. 6 Variation of Mean Flow Time with np (VLM)

Table V: System performance in VBM manufacturing environment.

\begin{tabular}{|c|c|c|c|c|c|c|c|c|c|}
\hline & \multicolumn{8}{|c|}{ Performance measure when number of pallets released to system } \\
\hline & & \multicolumn{2}{|c|}{6} & \multicolumn{2}{|c|}{8} & \multicolumn{2}{|c|}{10} & \multicolumn{2}{|c|}{12} \\
\hline & & Makespan & MFT & Makespan & MFT & Makespan & MFT & Makespan & MFT \\
\hline \multirow{6}{*}{ 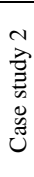 } & SPT & 11194 & 357.44 & 11237 & 471.88 & 10236 & 527.46 & 10608 & 616.39 \\
\hline & PT2 & 11426 & 358.43 & 10670 & 478.37 & 11120 & 532.22 & 10371 & 655.30 \\
\hline & \%CW & 11215 & 360.13 & 11127 & 475.36 & 12425 & 586.73 & 10133 & 715.96 \\
\hline & CW1 & 11326 & 362.17 & 11065 & 472.51 & 10802 & 588.96 & 9790 & 680.49 \\
\hline & CW2 & 11313 & 358.23 & 11145 & 473.17 & 11878 & 614.93 & 10683 & 666.33 \\
\hline & CW5 & 11227 & 358.17 & 10975 & 472.18 & 13441 & 568.95 & 13661 & 712.12 \\
\hline \multirow{6}{*}{ 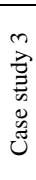 } & SPT & 8423 & 325.97 & 7806 & 426.46 & 8225 & 540.43 & 8732 & 618.29 \\
\hline & PT2 & 7649 & 331.13 & 7564 & 424.50 & 7674 & 516.37 & 8447 & 612.36 \\
\hline & \%CW & 7964 & 328.13 & 7752 & 428.62 & 10114 & 571.32 & 9813 & 701.62 \\
\hline & CW1 & 7979 & 330.42 & 7672 & 430.73 & 8354 & 525.70 & 8293 & 656.02 \\
\hline & CW2 & 8323 & 330.14 & 7785 & 431.67 & 9820 & 597.29 & 9415 & 676.43 \\
\hline & CW5 & 8267 & 327.71 & 7802 & 427.62 & 10114 & 572.11 & 9892 & 706.02 \\
\hline \multirow{6}{*}{ 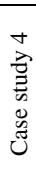 } & SPT & 17777 & 568.29 & 16119 & 618.02 & 15656 & 759.03 & 16113 & 915.21 \\
\hline & PT2 & 18984 & 591.16 & 15755 & 647.17 & 16815 & 854.48 & 16978 & 954.55 \\
\hline & \%CW & 18098 & 575.67 & 16200 & 630.13 & 13892 & 749.32 & 13153 & 918.47 \\
\hline & CW1 & 17876 & 570.62 & 15975 & 627.72 & 13015 & 776.98 & 16339 & 1012.44 \\
\hline & CW2 & 18975 & 580.17 & 16423 & 633.43 & 16264 & 879.01 & 15993 & 1003.89 \\
\hline & CW5 & 17795 & 577.32 & 15968 & 621.13 & 13738 & 751.95 & 15758 & 920.42 \\
\hline \multirow{6}{*}{ 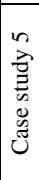 } & SPT & 11950 & 420.62 & 11688 & 447.41 & 10105 & 530.52 & 9871 & 575.17 \\
\hline & PT2 & 11805 & 410.03 & 12327 & 438.90 & 10213 & 522.35 & 9442 & 617.57 \\
\hline & \%CW & 11815 & 422.13 & 11700 & 450.13 & 10293 & 536.59 & 9084 & 646.81 \\
\hline & CW1 & 11825 & 412.72 & 11823 & 440.14 & 9760 & 548.75 & 9047 & 629.85 \\
\hline & CW2 & 11952 & 421.31 & 11915 & 450.47 & 10083 & 552.34 & 9497 & 673.05 \\
\hline & CW5 & 11940 & 418.71 & 12227 & 448.63 & 9447 & 573.35 & 10033 & 612.94 \\
\hline \multirow{6}{*}{ 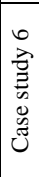 } & SPT & 7827 & 281.32 & 7774 & 300.69 & 7646 & 380.01 & 7652 & 453.93 \\
\hline & PT2 & 7787 & 285.84 & 7759 & 317.37 & 7732 & 379.96 & 7897 & 467.36 \\
\hline & \%CW & 7821 & 281.62 & 7773 & 310.01 & 9137 & 406.74 & 8615 & 493.50 \\
\hline & CW1 & 7835 & 285.73 & 7760 & 302.32 & 8734 & 412.76 & 8045 & 475.21 \\
\hline & CW2 & 7817 & 283.27 & 7780 & 312.52 & 8546 & 437.50 & 8325 & 512.64 \\
\hline & CW5 & 7822 & 284.72 & 7780 & 305.56 & 9007 & 444.79 & 8164 & 490.00 \\
\hline
\end{tabular}


Table V (Contd.): System performance in VBM manufacturing environment.

\begin{tabular}{|c|c|c|c|c|c|c|c|c|c|}
\hline & \multicolumn{8}{|c|}{ Performance measure when number of pallets released to system } \\
\hline & & \multicolumn{2}{|c|}{14} & \multicolumn{2}{|c|}{16} & \multicolumn{2}{|c|}{18} & \multicolumn{2}{|c|}{20} \\
\hline & & Makespan & MFT & Makespan & MFT & Makespan & MFT & Makespan & MFT \\
\hline \multirow{6}{*}{ 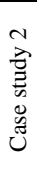 } & SPT & 10537 & 746.69 & 10803 & 832.18 & DL & DL & DL & DL \\
\hline & PT2 & 9499 & 757.48 & DL & DL & DL & DL & DL & DL \\
\hline & $\% \mathrm{CW}$ & 10457 & 850.92 & 9288 & 910.90 & 10719 & 1044.54 & $\mathrm{DL}$ & DL \\
\hline & CW1 & 9668 & 813.52 & DL & DL & DL & DL & DL & DL \\
\hline & CW2 & 10287 & 815.46 & 10539 & 896.44 & DL & DL & DL & DL \\
\hline & CW5 & 11373 & 899.69 & 11408 & 913.21 & DL & DL & DL & DL \\
\hline \multirow{6}{*}{ 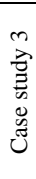 } & SPT & 8310 & 754.10 & 7837 & 899.12 & DL & DL & DL & DL \\
\hline & PT2 & 8407 & 741.42 & DL & DL & DL & DL & DL & DL \\
\hline & \%CW & 7984 & 769.59 & DL & DL & $\mathrm{DL}$ & $\mathrm{DL}$ & DL & DL \\
\hline & CW1 & 7966 & 812.42 & 7629 & 903.17 & DL & DL & DL & DL \\
\hline & CW2 & 8615 & 858.86 & DL & DL & DL & DL & DL & DL \\
\hline & CW5 & 8666 & 820.68 & 7653 & 876.71 & DL & DL & DL & DL \\
\hline \multirow{6}{*}{ 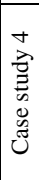 } & SPT & 14638 & 1060.95 & 14800 & 1200.82 & DL & DL & DL & DL \\
\hline & PT2 & 14660 & 1109.53 & 14458 & 1186.19 & 13340 & 1233.75 & DL & DL \\
\hline & \%CW & 13836 & 1036.5 & 12974 & 1181.69 & 13553 & 1306.67 & DL & DL \\
\hline & CW1 & 14821 & 1152.94 & 13191 & 1200.25 & 13568 & 1325.66 & DL & DL \\
\hline & CW2 & DL & $\mathrm{DL}$ & 14294 & 1279.55 & 13670 & 1361.94 & DL & DL \\
\hline & CW5 & 13928 & 1119.94 & 15328 & 1319.28 & 14729 & 1430.73 & DL & DL \\
\hline \multirow{6}{*}{ 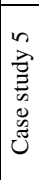 } & SPT & 9217 & 680.23 & 9007 & 783.50 & 9227 & 952.08 & 9137 & 1012.97 \\
\hline & PT2 & 9394 & 677.09 & DL & DL & 9275 & 865.39 & DL & DL \\
\hline & $\% \mathrm{CW}$ & 9598 & 701.45 & DL & DL & DL & DL & DL & DL \\
\hline & CW1 & 9180 & 670.60 & 9085 & 800.78 & DL & DL & DL & DL \\
\hline & CW2 & 9957 & 704.63 & DL & DL & DL & DL & DL & DL \\
\hline & CW5 & 9419 & 722.32 & DL & DL & DL & DL & DL & DL \\
\hline \multirow{6}{*}{ 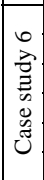 } & SPT & 7456 & 501.64 & 7643 & 545.05 & 7609 & 672.01 & DL & DL \\
\hline & PT2 & 7642 & 508.38 & 7392 & 583.33 & 7564 & 683.14 & DL & DL \\
\hline & \%CW & 8291 & 546.83 & 7647 & 690.59 & 7565 & 779.10 & DL & DL \\
\hline & CW1 & 7951 & 578.56 & 7729 & 612.23 & 7358 & 716.47 & 7513 & 820.77 \\
\hline & CW2 & 8118 & 603.98 & 7808 & 682.54 & DL & DL & DL & DL \\
\hline & CW5 & 8197 & 564.32 & 7582 & 697.02 & 7847 & 776.99 & DL & DL \\
\hline
\end{tabular}

Legend: $\quad$ MFT - Mean Flow Time $\quad$ DL - Deadlock State of System

Table VI: System performance in VLM manufacturing environment.

\begin{tabular}{|c|c|c|c|c|c|c|c|c|c|}
\hline & & \multicolumn{8}{|c|}{ Performance measure when number of pallets released to system } \\
\hline & & \multicolumn{2}{|c|}{6} & \multicolumn{2}{|c|}{8} & \multicolumn{2}{|c|}{10} & \multicolumn{2}{|c|}{12} \\
\hline & & Makespan & MFT & Makespan & MFT & Makespan & MFT & Makespan & MFT \\
\hline \multirow{6}{*}{ 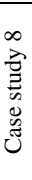 } & SPT & 1770 & 87.35 & 1759 & 119.33 & 1754 & 151.02 & 1754 & 169.68 \\
\hline & PT2 & 1767 & 86.91 & 1758 & 128.79 & 1755 & 152.92 & 1754 & 175.11 \\
\hline & $\% \mathrm{CW}$ & 1767 & 88.73 & 1760 & 120.12 & 1756 & 150.72 & 1755 & 170.52 \\
\hline & CW1 & 1764 & 87.43 & 1763 & 122.73 & 1752 & 151.63 & 1754 & 173.61 \\
\hline & CW2 & 1770 & 88.64 & 1758 & 121.14 & 1751 & 152.71 & 1756 & 172.21 \\
\hline & CW5 & 1772 & 87.77 & 1762 & 126.52 & 1754 & 152.72 & 1754 & 173.42 \\
\hline \multirow{6}{*}{ 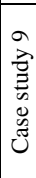 } & SPT & 1963 & 86.00 & 1843 & 113.33 & 1823 & 145.24 & 1820 & 158.57 \\
\hline & PT2 & 1986 & 88.83 & 1857 & 114.16 & 1823 & 145.22 & 1824 & 159.94 \\
\hline & \%CW & 1970 & 86.72 & 1845 & $\begin{array}{l}113.73 \\
\end{array}$ & 1824 & 146.01 & 1824 & 158.12 \\
\hline & CW1 & 1965 & 87.67 & 1855 & 115.21 & 1821 & 144.89 & 1822 & 159.72 \\
\hline & CW2 & 1972 & 88.14 & 1852 & 112.79 & 1822 & 145.72 & 1822 & 158.02 \\
\hline & CW5 & 1984 & 86.72 & 1850 & 115.12 & 1824 & 145.12 & 1824 & 159.23 \\
\hline \multirow{6}{*}{ 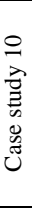 } & SPT & 2428 & 114.04 & 2424 & 160.11 & 2440 & 183.32 & 2419 & 216.04 \\
\hline & PT2 & 2437 & 115.31 & 2440 & 152.63 & 2449 & 169.57 & 2420 & 213.49 \\
\hline & \%CW & 2429 & 114.14 & 2426 & 160.00 & 2455 & 169.58 & 2418 & 217.73 \\
\hline & CW1 & 2430 & 115.12 & 2440 & 155.32 & 2460 & 169.12 & 2422 & 210.96 \\
\hline & CW2 & 2436 & 114.42 & 2432 & 158.71 & 2433 & 182.22 & 2420 & 216.79 \\
\hline & CW5 & 2433 & 114.63 & 2430 & 153.32 & 2493 & 169.42 & 2417 & 214.55 \\
\hline \multirow{6}{*}{ 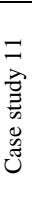 } & SPT & 1764 & 112.61 & 1762 & 135.72 & 1758 & 156.66 & 1734 & 196.44 \\
\hline & PT2 & 1758 & 114.84 & 1752 & 140.12 & 1748 & 161.27 & 1734 & 197.69 \\
\hline & \%CW & 1758 & 112.65 & 1753 & 140.03 & 1720 & 159.27 & 1723 & 201.01 \\
\hline & CW1 & 1764 & 113.42 & 1762 & 135.93 & 1721 & 167.29 & 1724 & 206.73 \\
\hline & CW2 & 1765 & 113.61 & 1761 & 138.72 & 1729 & 172.22 & 1737 & 191.41 \\
\hline & CW5 & 1754 & 114.32 & 1758 & 139.52 & 1723 & 173.01 & 1726 & 188.26 \\
\hline \multirow{6}{*}{ 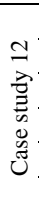 } & SPT & 2036 & 99.54 & 2025 & 122.32 & 2030 & 147.14 & 2019 & 161.67 \\
\hline & PT2 & 2031 & 100.31 & 2026 & 130.67 & 2026 & 156.71 & 2017 & 175.71 \\
\hline & \%CW & 2032 & 100.11 & 2025 & 123.12 & 2026 & 155.52 & 2019 & 161.23 \\
\hline & CW1 & 2031 & 99.73 & 2026 & 129.52 & 2026 & 148.12 & 2017 & 162.32 \\
\hline & CW2 & 2031 & 99.44 & 2025 & 126.12 & 2030 & 149.17 & 2018 & 169.12 \\
\hline & CW5 & 2033 & 99.62 & 2025 & 127.32 & 2030 & 156.22 & 2018 & 173.73 \\
\hline
\end{tabular}


Table VI (Contd.): System performance in VLM manufacturing environment.

\begin{tabular}{|c|c|c|c|c|c|c|c|c|c|}
\hline & & \multicolumn{8}{|c|}{ Performance measure when number of pallets released to system } \\
\hline & & \multicolumn{2}{|c|}{14} & \multicolumn{2}{|c|}{16} & \multicolumn{2}{|c|}{18} & \multicolumn{2}{|c|}{20} \\
\hline & & Makespan & MFT & Makespan & MFT & Makespan & MFT & Makespan & MFT \\
\hline \multirow{6}{*}{ 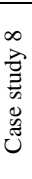 } & SPT & 1753 & 185.33 & 1750 & 208.26 & 1747 & 208.06 & 1747 & 257.09 \\
\hline & PT2 & 1751 & 198.12 & 1750 & 216.96 & 1747 & 238.69 & 1752 & 258.94 \\
\hline & $\% \mathrm{CW}$ & 1754 & 187.12 & 1752 & 215.62 & 1747 & 283.89 & 1745 & 316.19 \\
\hline & CW1 & 1754 & 196.23 & 1751 & 210.63 & 1747 & 281.62 & 1745 & 276.82 \\
\hline & CW2 & 1758 & 188.67 & 1750 & 212.12 & 1747 & 279.72 & 1745 & 316.19 \\
\hline & CW5 & 1754 & 192.42 & 1750 & 214.62 & 1747 & 262.42 & 1745 & 316.19 \\
\hline \multirow{6}{*}{ 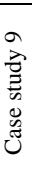 } & SPT & 1821 & 188.86 & 1820 & 215.16 & 1820 & 244.12 & 1817 & 264.38 \\
\hline & PT2 & 1821 & 184.83 & 1817 & 212.89 & 1817 & 237.66 & 1820 & 258.76 \\
\hline & \%CW & 1821 & 212.66 & 1815 & 251.44 & 1817 & 289.27 & 1820 & 308.66 \\
\hline & CW1 & 1821 & 208.42 & 1817 & 250.83 & 1816 & 259.49 & 1819 & 278.46 \\
\hline & CW2 & 1821 & 212.66 & 1820 & 249.78 & 1817 & 289.27 & 1820 & 308.66 \\
\hline & CW5 & 1825 & 213.03 & 1817 & 249.54 & 1817 & 288.93 & 1820 & 309.08 \\
\hline \multirow{6}{*}{ 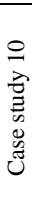 } & SPT & 2417 & 259.09 & 2420 & 272.83 & 2417 & 305.78 & 2417 & 333.06 \\
\hline & PT2 & 2420 & 244.74 & 2420 & 278.27 & 2420 & 305.28 & 2417 & 332.24 \\
\hline & \%CW & 2418 & 282.13 & 2417 & 308.32 & 2420 & 334.65 & 2420 & 383.07 \\
\hline & CW1 & 2420 & 246.02 & 2420 & 271.84 & 2420 & 296.67 & 2420 & 306.07 \\
\hline & CW2 & 2413 & 284.76 & 2413 & 317.65 & 2415 & 345.09 & 2416 & 381.09 \\
\hline & CW5 & 2417 & 274.19 & 2420 & 306.83 & 2420 & 337.14 & 2417 & 375.03 \\
\hline \multirow{6}{*}{ 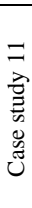 } & SPT & 1742 & 206.41 & 1754 & 218.97 & 1732 & 231.72 & 1732 & 245.67 \\
\hline & PT2 & 1740 & 208.41 & 1745 & 220.71 & 1740 & 235.54 & 1732 & 248.67 \\
\hline & \%CW & 1740 & 208.13 & 1754 & 219.93 & 1735 & 235.13 & 1732 & 246.12 \\
\hline & CW1 & 1743 & 206.73 & 1752 & 220.13 & 1738 & 233.72 & 1733 & 247.32 \\
\hline & CW2 & 1739 & 207.13 & 1746 & 218.99 & 1732 & 234.54 & 1732 & 245.73 \\
\hline & CW5 & 1742 & 206.84 & 1749 & 219.14 & 1739 & 233.16 & 1733 & 248.12 \\
\hline \multirow{6}{*}{ 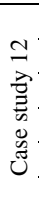 } & SPT & 2018 & 182.67 & 2015 & 215.67 & 2024 & 225.32 & 2021 & 268.67 \\
\hline & PT2 & 2019 & 195.25 & 2010 & 231.42 & 2026 & 241.27 & 2023 & 280.54 \\
\hline & \%CW & 2018 & 183.52 & 2015 & 216.32 & 2024 & 227.42 & 2021 & 267.67 \\
\hline & CW1 & 2018 & 186.13 & 2016 & 230.12 & 2024 & 230.61 & 2021 & 280.12 \\
\hline & CW2 & 2019 & 187.63 & 2017 & 229.42 & 2025 & 240.41 & 2023 & 275.42 \\
\hline & CW5 & 2017 & 186.63 & 2017 & 225.61 & 2024 & 235.42 & 2023 & 276.12 \\
\hline
\end{tabular}

A close observation of the system state at deadlock reveals that deadlock is reached due to non-availability of buffer space in the input buffer of next destination machine of the selected part i.e. deadlock occurs due to circular wait condition. It is important to mention that for the taken case studies in VLM (Table VI), system does not reach to deadlock state. This may be attributed to the fact that in VLM, operation times are comparable to transportation times. So by the time, R-2 transports a part to the input buffer of a machine, the machine completes the processing of under processed part. Since the input buffer capacity of the machine is three, it is very likely that there is always a vacancy in the input buffer of the machine. Thus, the parts available at B-IN and output buffer of each machine can be allocated to the next destination machine easily.

Table V also shows that for case study 2 in VBM, for PT2 and CW1rules at $n p=16,18$ and 20, system reaches to deadlock state and other values of $n p$ viz., $6,8,10,12,14$ do not give rise to deadlock state of PT2 rule. Similarly, for SPT, CW2 and CW5 rules, system reaches to deadlock state for $n p=18$ and $n p=20$ and other values of $n p$ do not give rise to deadlock state of system i.e. for a given production order and dispatching rule, deadlock state of system can be avoided by changing the $n p$. This observation is also seen for other case studies in VBM (Table V and Fig. 3). This phenomenon may be attributed to the fact that as $n p$ is increased from six to twenty in steps of two, shop load increases. The increase in shop load reduces the possibility of availability of buffer space in the input buffer of a machine. Thus, it may happen that the parts at B-IN and output buffer of each machine can not be allocated to their next destination machine according to the available flexible process plans as the input buffer of the next destination machine is full to its capacity. Thus, it can safely be concluded that for a given production order and DR, deadlock state of system can be avoided by changing the $n p$. 
Table V and Fig. 3 reveal that irrespective of DR used, no deadlock is observed for taken case studies in VBM when number of pallets released to the system is less than fourteen. This may be attributed to the fact that when number of pallets released to the system is less than fourteen, shop congestion is less and it is very likely that there is always a vacancy in the input buffer of the machine. Therefore, the parts at B-IN and output buffer of each machine can be allocated to their next destination machine according to their flexible process plans easily. Thus, it can safely be concluded that for a given production order, irrespective of DR, system does not reach to the deadlock state when number of pallets released to the system is less than fourteen.

This study also reveals some interesting aspects of system performance as measured by makespan and mean flow time. Table VII shows the impact of dispatching rule on makespan with minimum and maximum variations are shown in bold for VBM and VLM respectively. The impact of DR on makespan is computed as given below:

$$
\% \text { change in the makespan due to } \mathrm{DR}=\frac{\mathrm{Ma}_{2} \sim \mathrm{Ma}_{1}}{\mathrm{Ma}_{1}} \times 100
$$

where

$$
\mathrm{Ma}_{1}=\text { Minimum makespan that occurs for a DR }
$$

$\mathrm{Ma}_{2}=$ Maximum makespan that occurs for another DR

$\sim \quad$ = Difference

\begin{tabular}{|c|c|c|c|c|c|c|c|c|c|}
\hline \multirow{2}{*}{$\begin{array}{l}\text { Manufacturing } \\
\text { environment }\end{array}$} & \multirow{2}{*}{$\begin{array}{c}\text { Case } \\
\text { Study } \\
\text { No. }\end{array}$} & \multicolumn{8}{|c|}{ \%age change in makespan when number of pallets released is } \\
\hline & & 6 & 8 & 10 & 12 & 14 & 16 & 18 & 20 \\
\hline \multirow{6}{*}{ VBM } & 1 & 4.65 & 8.93 & 8.58 & 21.00 & 8.01 & 7.32 & 8.64 & $*$ \\
\hline & 2 & 2.07 & 5.31 & 31.31 & 39.54 & 19.72 & 22.83 & $* *$ & * \\
\hline & 3 & 10.12 & 3.20 & 31.80 & 19.28 & 8.79 & 2.73 & $*$ & $*$ \\
\hline & 4 & 6.79 & 4.24 & 29.20 & 29.08 & 7.12 & 18.14 & 10.41 & $*$ \\
\hline & 5 & 1.25 & 5.45 & 5.46 & 10.90 & 8.46 & 0.87 & 0.52 & $* *$ \\
\hline & 6 & 0.62 & 0.27 & 19.50 & 12.58 & 11.20 & 5.63 & 6.65 & $* *$ \\
\hline \multirow{6}{*}{ VLM } & 7 & 1.37 & 0.19 & 2.81 & 2.61 & 0.78 & 0.68 & 0.63 & 0.10 \\
\hline & 8 & 0.45 & 0.28 & 0.29 & 0.11 & 0.40 & 0.11 & 0.00 & 0.40 \\
\hline & 9 & 1.17 & 0.76 & 0.17 & 0.22 & 0.22 & 0.28 & 0.22 & 0.17 \\
\hline & 10 & 0.37 & 0.67 & 2.47 & 0.21 & 0.29 & 0.29 & 0.21 & 0.17 \\
\hline & 11 & 0.63 & 0.57 & 2.21 & 0.81 & 0.23 & 0.52 & 0.46 & 0.06 \\
\hline & 12 & 0.25 & 0.05 & 0.20 & 0.15 & 0.05 & 0.25 & 0.10 & 0.10 \\
\hline
\end{tabular}

Table VII: Effect of dispatching rule on makespan.

Legend: * - Effect can not be computed as all DRs give deadlock state.

** - Effect cannot be computed as all DRs except one give deadlock state.

Table VIII shows the effect of $n p$ on makespan with minimum and maximum changes are shown in bold for each manufacturing environment. The effect of $n p$ on makespan is computed as given below:

$$
\% \text { change in the makespan due to } \mathrm{np}=\frac{M a_{4} \sim M a_{3}}{M a_{3}} \times 100
$$

where

$$
\begin{aligned}
& \mathrm{Ma}_{3}=\text { Minimum makespan that occurs at one } n p \\
& \mathrm{Ma}_{4}=\text { Maximum makespan that occurs at another } n p
\end{aligned}
$$

Fig. 3 clearly reveals that in VBM, for a given $n p$, makespan is different for various DRs. This observation is also seen for other case studies in VBM and VLM (Fig. 5 and Tables V, VI). Table VII clearly reveals that variation in makespan due to DR is in the range of $0.275 \%$ to $39.54 \%$ for VBM and $0.10 \%$ to $2.47 \%$ for VLM respectively. This is due to the fact that 
different parameters are used by various DRs for part selection. Thus, it can safely be concluded that for a given production order and $n p$, makespan is dependent on DR used.

Table VIII: Effect of number of pallets released to system on makespan.

\begin{tabular}{|c|c|c|c|c|c|c|c|}
\hline Manufacturing & Case & \multicolumn{6}{|c|}{ \%age change in makespan when dispatching rule is } \\
\cline { 2 - 8 } environment & Study No. & SPT & PT2 & \%CW & CW1 & CW2 & CW5 \\
\hline \multirow{4}{*}{ VBM } & 1 & 35.97 & $\mathbf{4 7 . 0 3}$ & 41.95 & 41.53 & 39.41 & 34.87 \\
\cline { 2 - 8 } & 2 & 5.93 & 20.29 & 33.77 & 17.15 & 15.47 & 24.47 \\
\cline { 2 - 8 } & 3 & 12.25 & 11.67 & 30.47 & 9.50 & 26.14 & 32.16 \\
\cline { 2 - 8 } & 4 & 21.45 & 42.31 & 39.49 & 37.35 & 38.81 & 29.53 \\
\cline { 2 - 8 } & 5 & 32.67 & 32.91 & 30.06 & 30.71 & 25.88 & 29.81 \\
\cline { 2 - 8 } & 6 & $\mathbf{4 . 9 7}$ & 6.83 & 20.78 & 18.70 & 9.85 & 18.79 \\
\hline \multirow{4}{*}{ VLM } & 7 & 11.31 & 10.00 & 10.24 & 11.17 & $\mathbf{1 1 . 5 1}$ & 11.25 \\
\cline { 2 - 8 } & 8 & 1.32 & 1.15 & 1.26 & 1.09 & 1.49 & 1.55 \\
\cline { 2 - 8 } & 9 & 8.04 & 9.30 & 8.54 & 8.20 & 8.53 & 9.19 \\
\cline { 2 - 8 } & 10 & 0.95 & 1.32 & 1.57 & 1.65 & 0.83 & 3.14 \\
\cline { 2 - 8 } & 11 & 1.85 & 1.50 & 2.21 & 2.50 & 2.08 & 2.03 \\
\hline
\end{tabular}

Fig. 3 also reveals that in VBM, for a given DR, makespan varies as $n p$ is varied from six to twenty in steps of two. This observation is also seen for other case studies in VBM and VLM (Fig. 5 and Tables V, VI). Table VIII reveals that variation in makespan due to $n p$ is in the range of $4.97 \%$ to $47.03 \%$ for VBM and $0.69 \%$ to $11.51 \%$ for VLM respectively. This phenomenon may be attributed to the fact that as the number of pallets released to the system is increased, shop load increases. Thus, it can safely be concluded that for a given production order and dispatching rule, makespan is dependent on $n p$.

Figs. 4 and 6 clearly show that as $n p$ is increased from six to twenty in steps of two, irrespective of dispatching rule, mean flow time (MFT) increases in VBM as well as in VLM. This observation is also seen for other case studies in VBM and VLM environment (Tables V, VI). This phenomenon may be attributed to the fact that as $n p$ is increased in the taken range, shop load increases. Thus, it can safely be concluded that in the considered manufacturing environments, as $n p$ is increased in the taken range, MFT increases gradually and minimum MFT in each environment is achieved by releasing six pallets to the system.

\section{CONCLUSIONS}

This paper investigates the performance of an example FMS in VBM and VLM environments in the presence of FPPs from deadlock, makespan and mean flow time viewpoints. The following conclusions are drawn from the simulation results for the taken case studies.

(i) Deadlock state of system can be avoided either by changing the dispatching rule or number of pallets released to the system.

(ii) The system will not reach to deadlock state, irrespective of dispatching rule, when number of pallets released to the system is less than fourteen.

(iii) For a given production order, makespan is dependent on number of pallets released to the system. Variation in makespan is in the range of $4.97 \%$ to $47.03 \%$ in VBM and $0.69 \%$ to $11.51 \%$ in VLM respectively.

(iv) Choice of dispatching rules is important in FMS and affects the system performance. The variation in makespan is in the range of $0.27 \%$ to $39.54 \%$ in VBM and $0.10 \%$ to $2.47 \%$ in VLM respectively. This conclusion is in line with previous study [25, 31].

(v) In each manufacturing environment (i.e. VBM and VLM), as the number of pallets released to the system is increased from six to twenty in steps to two, mean flow time 
increases gradually. Thus, six numbers of pallets should be released (minimum number considered in the present study) in order to have minimum flow time.

\section{REFERENCES}

[1] MacCarthy, B. L.; Liu, J. (1993). Addressing the gap in scheduling research: a review of optimization and heuristic methods in production scheduling, International Journal of Production Research, Vol. 31, No. 1, 59-79

[2] Blazewicz, J.; Finke, G.; Haupt, R.; Schmidt, G. (1988). New trends in machine scheduling, European Journal of Operational Research, Vol. 37, 303-317

[3] Van Looveren, A. J.; Gelders, L. F.; Van Wassenhove, L. N. (1986). A review of FMS planning models, Andrew Kusiak (Editor), Modeling and Design of Flexible Manufacturing Systems, Elsevier Science Publishers, Amsterdam, 3-31

[4] Ishii, N.; Talavage, J. J. (1991). A transient based real time scheduling algorithm in FMS, International Journal of Production Research, Vol. 29, No. 12, 2501-2520

[5] Yang, Z.; Qiao, L.; Jiang, L. (1998). Improving the performances of part dispatching based on multiple process plans using graph theory, International Journal of Production Research, Vol. 36, No. 7, 1987-2003

[6] Benjaafar, S.; Ramakrishnan, R. (1996). Modeling, measurement and evaluation of sequencing flexibility in manufacturing systems, International Journal of Production Research, Vol. 4, No. 5, 1195-1220

[7] Hutchinson, G. K.; Pflughoeft, K. A. (1994). Flexible process plans: their value in flexible automation systems, International Journal of Production Research, Vol. 32, No. 3, 707-719.

[8] Coffman, E. G.; Elphick, M. J.; Shoshani, A. (1971). System deadlocks, ACM Computing Surveys, Vol. 3, No. 2, 67-78

[9] Kundu, S.; Akyildiz, I. F. (1989). Deadlock free buffer allocation in closed queuing networks, Queuing Systems, No. 4, 47-56

[10] Koh, I.; DiCesare, F. (1992). Transformation methods for generalized Petri nets and their application to flexible manufacturing systems, Proceedings of Rensselaer's Second International Conference on Computer Integrated Manufacturing, Troy, New York, May 20-22, 364-371

[11] Zhou, M. C.; DiCesare, F. (1990). Parallel and Sequential mutual exclusions for Petri nets modeling for manufacturing systems with shared resources, IEEE Transactions on Robotics and Automation, Vol. 6, 515-525

[12] Wysk, R. A.; Yang, N. S.; Joshi, S. (1994). Resolution of deadlocks in Flexible manufacturing systems: Avoidance and recovery approaches, Journal of Manufacturing Systems, Vol. 13, No. 2, 128-138

[13] Banaszak, Z. A.; Krough, B. H. (1990). Deadlock avoidance in flexible manufacturing systems with concurrently competing process flows, IEEE Transactions on Robotics and Automation, Vol. 6, No. 6, 724-734

[14] Viswanadham, N.; Narahari, Y.; Johnson, T. L. (1990). Deadlock prevention and deadlock avoidance in flexible manufacturing systems using Petri nets model, IEEE Transactions on Robotics and Automation, Vol. 6, No. 6, 713-723

[15] Leung, Y. T.; Sheen, G. J. (1993). Resolving deadlocks in flexible manufacturing cell, Journal of Manufacturing Systems, Vol. 12, No. 4, 291-304

[16] Vishwanadham, N.; Narahari, Y. (1992). Performance Modeling of Automated Manufacturing Systems, Prentice-Hall, New Delhi

[17] Desrochers, A. A.; Al-Jaar, R. Y. (1994). Applications of Petri Nets in Manufacturing Systems, IEEE Press, New York

[18] Dijkstra, E. W. (1968). Co-operating sequential processes, F. Genuys (Editor) Programming Languages, Academic Press, London, 43-112

[19] Haberman, A. N. (1969). Prevention of system deadlocks, Communications of the ACM, Vol. 12, No. 7, 373-385

[20] Gaarder, E. (1993). Deadlock avoidance in flexible manufacturing systems, Master's Thesis, University of Illinois at Champaign-Urbana, IL 
[21] Lawley, M. (1995). Structural analysis and control of flexible manufacturing systems, Doctoral Thesis, University of Illinois at Champaign-Urbana, IL

[22] Lawley, M.; Mittenthal, J. (1999). Order release and deadlock avoidance interactions in counterflow system optimization, International Journal of Production Research, Vol. 37, No. 13, 30433062

[23] Lawley, M. (1999). Deadlock avoidance for production systems with flexible routing, IEEE Transactions on Robotics and Automation, Vol. 15, No. 3, 497-509

[24] Jain, A.; Jain, P. K.; Singh, I. P. (2003). Real time scheduling in FMS: suitability and effectiveness of flexible process plans, International Journal of Simulation Modelling, Vol. 2, No. 3, 57-69

[25] Jain, A.; Jain, P. K.; Singh, I. P. (2004). An investigation on the performance of dispatching rules in FMS scheduling, International Journal of Simulation Modeling, Vol. 3, No. 2-3, 49-60

[26] Caprihan, R.; Wadhwa, S. (1997). Impact of routing flexibility on the performance of an FMS- a simulation study, International Journal of Flexible Manufacturing Systems, Vol. 9, 273-298

[27] Weintraub, A.; Cormier, D.; Hodson, T.; King, R.; Wilson, J.; Zozom, A. (1999). Scheduling with alternatives: a link between process planning and scheduling, IIE Transactions, Vol. 31, 1093-1102

[28] Borenstein, D. (2000). Implementation of an object-oriented tool for the simulation of manufacturing systems and its implementation to study the effects of flexibility, International Journal of Production Research, Vol. 38, No. 9, 2125-2142

[29] Gan, P. Y.; Lee, K. S. (2002). Scheduling of flexible-sequenced process plans in a mould manufacturing shop, International Journal of Advanced Manufacturing Technology, Vol. 20, No. 3, 214-222

[30] Jain, A. K.; Elmaraghy, H. A. (1997). Production Scheduling/Rescheduling in Flexible Manufacturing, International Journal of Production Research, Vol. 35, No. 1, 281-309

[31] Lee, D. H.; Kim, Y. D. (1996). Part-mix allocation in a hybrid manufacturing system with flexible manufacturing cell and a conventional job shop, International Journal of Production Research, Vol. 34, No. 5, 1347-1360 